\title{
Technologies that through Synergic Development can support the Intelligent Automation of Business Processes
}

\author{
Vasile MAZILESCU ${ }^{\star}$, Adrian MICU ${ }^{\star \star}$
}

\begin{tabular}{l}
\hline \multicolumn{1}{c}{ A R T I C L E I N F O } \\
\hline Article history: \\
Accepted June 2019 \\
Available online August 2019 \\
\hline JEL Classification \\
M10, M15 \\
Keywords: \\
DT, Business Process \\
Automation/Robotization (BPA/R), \\
AI, InA, CTs, Web4.0
\end{tabular}

\section{Introduction}

DT seems a difficult task for organizations that have not made progress yet. The concept of digitization targets multiple aspects at the level of a company and at the level of internal and external processes, such as the process of converting at one company level any analog information or documents, images, photographs, sounds, signals, location data, identity cards, etc. in digital format for use through computerized data storage systems, platforms, applications $[4,9]$. While InA focuses on automating human physical work (for example, assembling products), BPA/R is the automation of work / business flows to make decision-making more efficient at any level that, with the passage of time, becomes complicated, inefficient and costly [3,8,17]. BPA / $\mathrm{R}$ can be a cornerstone for adopting that culture of continuous DT, being an essential element of any modern business. It increases the efficiency and productivity of each job. At present, businesses are increasingly turning to $\mathrm{BPA} / \mathrm{R}$ to assist in making decisions, providing recommendations or delivering services, that is, economic activities through which providers and customers exchange benefits $[11,24,26]$. These benefits can be objects of economic value, such as money or other physical goods. The business field can be used extensively by InA-based technologies, especially in marketing, finance, logistics, e-commerce, and services. Business processes are often expressed through the synthesis of digital service packages $[7,18]$. A service package is made using Web services as business processes. Web services are software applications used on the Internet. Based on increasing, unstructured data, business processes implemented in the form of services can benefit from AI technologies that enable decision-makers to assist, draw action rules, reuse agent-based knowledge [33]. AI is a key technology whose importance can not be neglected, being a global innovation that will profoundly change the economy, politics and society $[2,12,48]$. Classical computer information systems must be programmed (using different procedures, conceptual models, programming languages) and work in a procedural manner, performing only predefined functions. AI systems are characterized by the ability to learn and develop on themselves and can be used in a wide range of difficult or routine tasks [1,5,40]. AI systems are developed to be used in the control of industrial installations, in the field of medical diagnosis, investment 
consultancy, supervision of insurance contracts, lawyers' activities, law enforcement, cyber security, military, etc. $[6,13]$. Industry 4.0 is an extremely active domain and discussed and falls within the context of this work because it is characterized by a massive process of InA. It is obviously a major example of application and business for the whole range of technologies analyzed in this paper. Industry 4.0 is not possible without Big Data, AI and Cloud Computing, along with many other highly visible technologies currently on the market $[14,16]$. Companies need to use the opportunities offered by AI-based technologies to further develop their business models, even though some of these intelligent technologies are still in the process of maturing [21]. The AI domain is not new in computer science, with a more or less satisfactory evolution in relation to the capital and human investments made $[10,20]$. The fact is that AI is a priority area of application research and development globally. There are nuances regarding the involvement of different states in this area. China will soon become, together with USA, a global leader in AI and CTs, with more and more consistent investments being made. Using different decisional models, CTs add traditional business management information systems higher intelligence chracteristics: learning, discovery, adaptation, reasoning, sentiment analysis [31,37]. CTs are the next generation information systems that are capable of understanding, making appropriate judgments, interacting continuously with the environment or other agents (generating this way knowledge), understanding natural language and interacting with heterogeneous agents. These technologies must integrate intelligent capabilities such as Automated Learning (ML) and Deep Learning (DL), generate results based on descriptive and predictive analytics, support the automation of various business processes, contribute to the innovative transformation of chains of value creation [46]. The use of assisted learning in user-centered IoT devices is a step forward in their communication. The main benefit that intelligent agents bring to IoT devices is the creation of new systems based on user needs. IoT devices have experienced a massive development in the last years, and interconnected objects have begun to provide a lot of data about what they are being applied to. However, data is not a value unless it is transformed into information that may be useful later. This refers to the Big Data domain through which raw data is processed to obtain valuable information. However, it takes time and study to have knowledge about what has been done or what is being done. CTs need to be focused on analyzing data and synthesizing recommendations according to customer interests and expectations, using statistical and ML algorithms to achieve planning and prediction functions, learn autonomously from their own experience and be able to explain the results (similar with Truth Maintanace Systems in Advanced Expert Systems), understand natural language. These systems can really contribute to increasing all kind of communication strategies, can simulate human intelligence functions as close as possible to human experts, develop analysis and action capabilities [45].

This paper presents in Section 2 an analysis of DT in the context of unprecedented growth of AI capabilities to support new computational systems to automate work in a natural way. All economic sectors are affected by DT, both for the production of goods and for the development and delivery of services. Business models need to be reviewed in this context as well as how key technologies presented can provide opportunities for sustainable development. In Section 3, the AI features are outlined in the different forms of reasoning and representation of knowledge. There are described in Section 4 the transformation technologies of the CTs type, that is, those software systems synthesized in the form of agents that operate autonomously and can make intelligent decisions in complex situations using automated reasoning. All of these technologies will be gradually integrated into Web4.0 and even Web5.0, with profound implications for companies and the entire business environment. Section 5 looks at a range of technologies that are the real support for InA. If for the industrial field this has been done successfully for a long time, BPA is also contemplated recently, given that different technology mixes allow for real-time activities with a bigger degree of complexity. At the end of the paper, we try to highlight a series of conclusions regarding the development and implementation of systems that allow the efficient deployment of InA in the context of global efforts to give computers the capabilities that experts have in their various areas using CTs.

\section{DT premise for the development of smart and efficient technologies}

DT assumes the use of digital services at the main important business processes level: ERP solutions (designed for integrated management of all processes and operations within a company within a single IT platform), CRM solutions (a set of tools, procedures and strategies that have customer relationship improvement), ARP (Advanced Planning \& Scheduling for Production Planning) solutions, Business Intelligence solutions for data analysis, mobile digital applications for sales team management, e-commerce sales (selling products through an online platform - virtual stores), etc. [25,30]. It transforms business models, allows obtaining new production processes and the development of new services such as eGovernment, blockchain and, implicitly, electronic payments. DT naturally and functionally involves the intense collaboration between competing companies to build complex ecosystems, bringing change not only in the area of consumers and sales but also in the producers and developers, which in some cases turn from competitors to collaborators. This way DT ensures interoperability of products to strengthen the new ecosystem of existing devices and applications [43]. Major IT companies need to work together to put in evidence the product compatibility. This collaboration is, however, limited to the interfaces of the relationships between them, while the companies remain competing. With the massive DT of the companies, 
the dynamics of the application industry will totally change other industries. DT will change the entire business ecosystem that is bound to follow new trends. If at the top level of companies, digitization leads to profit and new opportunities, at the bottom level, digitization can bring about a change in the company's structures and cost. A company that recommends customers what to do in the human resources field is totally different from one that only sells products or service packages. A coherent DT is achieved by opening new possibilities from the perspective of users, not by imposing them on the strict use of some products. Thus, the entire business ecosystem is gradually transforming, moving from product orientation to consumer orientation and preferences $[44,49]$. The definition of strict ecosystems imposed by companies will change altogether through the pressure of customers, who will avoid the products that limit them only to their purchase. Traditional companies will have to adapt their vision, copying the model adopted by former competitors, which are now undertaking various collaborative processes that have been impossible to imagine until a few years ago. Technologies based on the intelligent representation and processing of data and knowledge have a decisive role in the ample DT, ranging from defensive strategies to innovation-oriented strategies and increasing business process efficiency and resource utilization. DT at a company level can be applied for distribution, sales, financial, production, communication, etc., as well as for the transfer of administrative or promotional and marketing practices (electronic invoicing, electronic signature, electronic archiving, cloud). Intelligent DT wiil affect every company and involves the use of AI-based technology integrated on different new platforms, being in a continuously conceptual and practical process. It is based on the implementation of services and service packages very close to the needs of consumers [19,21]. In the context of DT, organizations and people need to adapt to meet the new opportunities. Depending on industry and activity centered on products or services, the impact of DT differs. The large amount of data to be converted into information allows companies to diagnose, and at that time can optimize a number of processes or decisions. Capitalizing on these data and turning them into extremely useful information and even knoeldge with business analytics helps companies better segment communication, better target goals, and deliver relevant messages to consumers. Every consumer leaves an own fingerprint. Recognition of the power and value of these data is possible with the help of people and AI technologies that have the ability to interpret and use them. Intelligent DT is not so much about what a company does, how it does for the benefit of consumers and the company. DT's benefits are universally accepted and summarize the reduction of labor costs in the context of a crisis in this area. It also allows the improvement and simplification of communication with customers and suppliers, increasing the visibility of companies in the online environment through more and more intelligent platforms (standardized hardware and software reconfigurable structures, with network effects at the same time, which leads to cost savings and a difficult quantification of the digital competition), increased accessibility to information of interest to companies, more efficient management of documents and inventories, improving internal quality standards by optimizing monitoring processes, increasing sales, man-machine communication in a semantic way [38,39].

\section{Current AI features}

AI is currently a technological trend because it offers new opportunities for different areas. Using these technologies, computers learn to solve specific tasks by processing a large amount of data and recognizing some forms. The technological evolution of recent years influences decisively all existing industries. AI aims to give computers a series of capabilities to accomplish tasks that the human decisionmaker is able to do with symbolic approaches based on logical formalisms and neural network-based connections. Connectionist systems support the solving of complex problems such as understanding natural language, form recognition, process management, classification, etc. [32,36,50]. Neural networks try to mimic the way the human brain works by creating connections between processing elements called neurons (formal). Artificial Neural Networks (ANN) are part of the AI domain, being able to describe a problem and solve it in a very short time without using a particular program. Solving the problem involves a selforganizing of the ANN following a learning process. Thus, a number of training networking algorithms have been developed, each of which has advantages and disadvantages and can be successfully applied in some cases. ANN have learning, synthesis and generalization capabilities. To create a neural network it is necessary to know the data on the input layer and the data on the output layer. The inputs of an artificial neuron are numerical values from the set of real numbers that can be represented as n-matrix and a single column. Each element in the array is associated with an exciting weight (positive numerical values) or inhibitory weight (negative numerical values). At the sum of the weighted inputs we add a polarization term, which expresses the initial state of that neuron. The term polarization is found in the literature as the bias. The value of the weighted input values and the bias term uses an activation function, resulting in the output of the neuron. The most important stage in the process of designing and building of any neural network is learning. It involves a change in the weights of specific connections between neurons of the neural network, a modification made to encode all information. The learning process involves a set of data known for training and an algorithm capable of adapting to the information received. Learning in this context refers to the different techniques and ways in which a machine or computer learns (ML) to perform a certain task and to do better without being explicitly programmed for it. Deep learning (DL) is a subset of ML algorithms that are characterized in that it first learns how to process input data and then the desired function. DL algorithms do not require extraction of 
category features in advance because they learn to do so automatically. Today we are interested in standardizing work processes and replacing activities with robots and intelligent machines instead of people who will have to turn to new areas. We cannot speak of systems as intelligent as humans, but of highly focused AI applications and models on specific types of issues, including ML and DL. ML is committed to developing the learning capabilities of software systems. They use algorithms that identify data models that can be used to make business decisions or predictions [15]. ML is a subset of AI that focuses on building systems that can learn - or improve performance based on data they process. Today there are ML-based applications that work virtually all around us [27]. When we interact with banks, buy online or use social media, ML algorithms come into play to create an effective, smooth and secure experience. ML and the technology that supports it grows fast, although we are only beginning to use them in concrete and meaningful applications. ML platforms efficiently exploit AI methods and integrate different functionalities within them. They are cloud solutions that build mathematical models that will later be used in application development. Currently, their primary goal is to implement predictive analysis solutions. DL offers computers the ability to think, learn as people are doing. This branch uses a complex architecture in which the natural neural networks are replicated, thus contributing significantly to the personalization of the experience [46]. Companies will have employees to monitor and guide neural networks, even using voice recognition. The process of converting words into digital format for the purpose of transcribing them into a code useful for various software applications is called voice recognition. It is mainly used for interactive voice response and mobile applications. Language technology and natural language processing include systems that use a lot of models and methods for textual analysis. In order to get a good understanding of the content both in terms of syntax and semantics, these systems use technologies to identify the vocal tone and the intensity of expression of a sentence [22].

The study of reasoning processes and intelligent behavior is about understanding, representing and solving problems, all of them being the symbolic component of AI field. It was initiated in 1956 by John McCarthy, Marvin Minsky, Allen Newell and Herbert Simon of Dartmouth College, aiming to formalize intelligent action. A rigorous definition of symbolic AI is based on a series of concrete knowledge of this discipline and especially on its logical foundations. Logic is a much earlier discipline as AI that has developed for reasons and objectives different from AI-specific ones. Mathematical logic was formed at the beginning of the last century to answer a series of fundamental mathematical problems, the initial objective being to research the notion of calculability and demonstration. The basic idea of logic is the study of reasoning in various formal theories, which is why symbolic AI is based on these formalisms. The model is considered as a main concept of the first order language semantic. Model theory aims at describing models of axiomatic theories in order to highlight their mathematical structures (algebraic, topological). In the case of the theory of demonstration, are studied problems of the complexity of demonstrations for the same theorem, according to different deductive systems or languages (regularity, equivalents, similarities). The calculability theory studies the intrinsic notion of computational function with its various models: the Turing machine, recursive functions and combinatorial logic with a number of properties. Starting from these notions can be studied the concept of decidability, accompanied by a study of various models to demonstrate the decidability or nondecidability of mathematical theories. The theory of abstract complexity, which generally uses different variants of the Turing machine (as an elementary calculation model for evaluating the computational resources needed to solve given problems), allows the establishment of complexity classes for decidable problems. We need to emphasize the unity of these three major issues. The concepts of computation and demonstration are closely linked, and precise correspondences can be established between them. Model theory can contribute to deciding issues and can provide semantic justification of inference rules and automatic demonstration systems. This unit is an essential feature of logic, which is a theoretical reference body. From the perspective of the symbolic AI, there are found the specific tendencies of the three characteristics presented above: semantics and model theory, demonstration and reasoning, calculus and complexity, but with often distinct modalities in relation to theories specific to mathematical logic alone. Real-time computing is an area of intense research in AI field, since the correctness of a system operating in a dynamic and distributed environment is not only dependent on the logic of its realization, but also on the temporal aspects involved [35]. Such systems also include AI agents subject to various complex time constraints, comprising different levels of time granularity. Timing knowledge is an essential aspect for a large number of AI applications (planning, process management, dynamic situation management, multi-agent systems). An intelligent agent must have reasoning capabilities that take into account a series of events that may occur in the real process: interruptions, limitations of processing times, synchronous or asynchronous character of the new information appearance. The time consideration should highlight two complementary aspects: the temporal information management and the formalization of time for real time reasoning. Some approaches are based on numerical models, and others on symbolic representations of time. Real-time reasoning has specific features: real-time operation often involves a temporal reasoning, but contrary this is not always true. Intelligent agent software solutions are used to prevent fraud attempts and are preferred by some companies in analyzing unstructured data such as online conversations or increasing virtual assistants' efficiency $[34,41]$. Agents are autonomous, adaptive software products that can be used to perceive the environment they come from. With the help of sensors and based on the knowledge from which they started, 
using assisted learning accumulates as much positive or negative knowledge about the environment in which they work. Based on the information they receive later, they can make their own decisions by putting them into action with the help of the effectors. Based on assisted learning the agents are helped to make decisions based on feedback received from the user or the system they come from, in the various emerging and unknown situations. After the agent has formed a knowledge base, the next occurrence of the learned situations will know what to do.

\section{We can use CTs to develop Web4.0}

$\mathrm{AI}$ is directly involved in the development of CTs, technologies that can emulate as accurately as possible the reasoning and the behavior of a man [28,29]. The important issues involved in these technologies can be classification, planning, diagnosis, learning, that is, all the representative and generic problems that AI is trying to solve in a traditional way. CTs are involved in Web browsing using cognitive models, neuromorphic calculus and devices, space-time events recognition, human reasoning mechanisms, expert systems and knowledge representation, adaptive and mobile multi-agent systems, collective intelligence and intelligent agent coordination, emotional modeling, ubiquitous systems, etc. Cognitive computing is at the heart of these technologies and is at the intersection of neuroscience, Big Data, Supercomputing and nanotechnology. The leader in CTs is the IBM company that proposes the Cognitive Enterprise model, focusing in the near future on Cognitive Enterprise [31]. These technologies have to automate those core business activities, with important consequences in gaining value and increasing productivity with the automated synthesis of company-specific service packages. All these concepts and technologies need to be integrated into business platforms to build intelligent business. Essential in this sense remain IoT, Blockchain, AI technologies.

CTs are closely related to semantic technologies and Knowledge Management (KM), KM being the initial of new knowledge generation process for both people and intelligent agents. Successful managers have always used intellectual goods and recognized their value. But these efforts have not been systematic, sharing knowledge has not always been done so that it will bring significant profit to organizations. The structuring of knowledge and associated processes with them, based on technologies, increasingly suited to the nature and interpretation of knowledge in various and varied applications (known as semantic technologies with embedded intelligence) allows effectively and efficiently problem solving, dynamic learning, strategic planning and taking decisions. Planning a business strategy is a good example, in which abstraction as a cognitive process must be correctly used. In this case, planning deals with concepts such as economic growth, tracking customer satisfaction, real resource allocation, policy synthesis. Numerical data purchased from various sources must be interpreted to provide a correct solution, a good plan. An intelligent agent performing this data interpretation is a valuable assistant in business processes that involves the synthesis of a business strategy. An intelligent planning agent needs a number of knowledge and must be able to carry out a series of logical operations on symbolic structures in order to obtain a correct solution, ie a plan or a good business strategy in the context of competition market. Solving a problem with a single knowledge-based system implies that this system embraces a general view of the problem and possesses all the reasoning capabilities and knowledge needed to solve it. In the case of complex problems, a single knowledge-based system (agent) is not able to solve the objectives of the problem in question, requiring the involvement of several systems (artificial or classical intelligence), each of which has only a partial view of the problem. In addition, these systems need to cooperate with each other to achieve the overall goal of the system. This is the case for multi-agent systems, their principle being sharing between multiple agents (intelligent or not) and sharing the knowledge and reasoning capability that the system possesses in its entirety. Each of these systems is specialized in solving a subproblem, in collaboration and continously communication with other system agents to improve their own participation in solving the problem globally and completing the information they may need. From a cognitive point of view, communication refers to aspects of perception, individual reasoning and action. All of these technologies are extremely important for Web 4.0. The ubiquitous computation refers to a new computing era, in which various computational elements such as mobile devices, electronic equipment, etc. will be integrated. The crucial element of ubiquitous computing will be the high level of communication between the different participating devices, which will allow for a sincronized, highly secure communication infrastructure. Web 4.0 is the same as the Web of Things (subset of the general concept of IoT), will be based on a universal web personality of each user, where the flow of information will be highly personalized. User anonymity will be impossible in Web 4.0, seen as a mobile space where users, real and virtual objects, based on an ontological description, are integrated together to create value. Current XML-based standards for describing web services provide a syntactic description of the service, while the semantics associated with it and the general semantics of the entire context of search and interpretation are lacking. For this reason, it is not possible to automatically interpret web services, and the automatic combination of web services is difficult. Solving these issues involves the use of ontologies, explicit formal specifications of concepts in a given field, and the relationships between them. An ontology defines a common vocabulary for a particular domain, each term being explicitly defined and possessing a semantic processable by the computer. Ontologies facilitate communication and provide access to content-based information. Semantic Web Services integrates classical web services ontologies to make it easier to identify, compose and automatically preserve web services. The 
ontology-based representation adds semantic information to the user's natural logical logic, using RDF / RDFS, OWL / OWL-S, WSMF / WSMO tools. It involves a set of knowledge, the semantic links between them and some logical inference rules. The Web Services Semantics provide a description of the services at the level of the implemented process that contains, besides functional information and other information such as the preconditions and post-conditions of the service operations, as well as the effects of executing a particular operation. To implement these semantic features at Web 3.0 and Web 4.0, it is necessary to define semantic metadata, specifically added to the description of the service, in such a way that by using this semantic information, an application can actually process the data necessary for a specific objective. Web 4.0 is intimately linked to the concept of Augmented Reality, as the evolution of this technology will enrich the real world with digital information and emotional sharing capabilities. Since people and businesses are mutually dependent, Web 4.0 has features of consensual and symbiotic functioning between employees and the business world. It is estimated to mature over the next decade and has the AI technology base and the technology of collectivities of connected and team-based agents (collaborative). It can integrate heterogeneous and highly distributed knowledge models, exploited by highly advanced algorithms. Of course, in this overall context Big Data will be important in identifying the various changes and trends of the business environment, which are extremely useful in the synthesis of strategies and plans of different companies. In the perspective, we can also discuss about Web 5.0 for which the various attributes still poorly drawn are also based on intelligence characteristics $[41,46,47]$.

\section{Intelligent processes Automation}

Maintaining competitiveness is close linked to the implementation of new technological innovations and process automation. BPA is one of the most powerful technology trends, as it can be applied to a large number of industries. Thanks to him, many human activities have been automated and business processes have been optimized. In the recent times - especially in the context of a major workforce and talent shortage more and more business process automation (BPA) is being talked about, and at the same time, more and more companies are implementing software products to automate at least some of the daily or regular activities $[3,8]$. BPA brings added value to any business by: increasing financial performance, allowing it to save time and money, rethinking business in a way more suited to the technological era we are in, and the rapid transformation the world has today, digitization of business activities and related processes in the future, discovering new market opportunities, identifying and optimizing business processes, making better business decisions, increasing predictive financial performance, etc. BPA can be used to simplify various processes such as document management, workflow automation, email alerts, e-mail marketing campaigns, thank-you letters, payment reminders, document review and approval, management of employees' requests. Popular areas of use include marketing, customer management, and customer support. By using software technology, the BPA strategy leads to the organization of staff and systems through efficient workflows, eliminating the need for employees to perform repetitive or low-level tasks, all of which are left behind by integrated applications. Automation enables the company to maintain control over various aspects such as customer relationships, analysis, planning, sales, standardization and development. Included are both complex IT tasks, such as system user management and network troubleshooting, as well as programs such as e-mail marketing services. BPA/R radically alters how we interact with and our position in society. It causes changes both personally and professionally, develops virtual shopping functionality, giving personalized recommendations and discussing purchasing options [10]. BPA systems dedicated to supply chain management and distribution are often integrated with marketing. For example, they ensure the smart distribution process by taking into account certain campaigns or promotional offers, and predict both the level of demand and consumer buying habits. Intelligent BPA/R is based on a big variety of business technologies that produce a dislocation in the usual course of business accompanied by major changes in traditional markets. The new economy puts the demand at the forefront, the needs of consumers who are increasingly involved in conceiving, realizing and using goods and services, starting from the stage of their research and development. From this point of view, the new economy has an interactive, participative character, making the interface between supply and demand in a volume and structure area, in space and time, much more rigorous. The role of the consumer grows especially in the sense that it can become an important source of innovative ideas for the producer or forcing innovation to maintain or expand the market, increase the level of comfort or, what is very important, increase the level of sustainability of economic development. Technologies may have different influences on the business [43]: transformational (with significant changes to the organization), high (with a high impact on work and people's lives), environment (influences business processes, services and people's lives) only certain processes and aspects of consumer life). From the point of view of maturity, technologies can be mainstream (there is a clear need and many clients implement different solutions), adopted early (clients are willing to adopt them), young (discussed by analysts and accepted by leaders), emerging researched and applied only on specialized markets). Unlike classical technologies that incrementally improved without overly disturbing the application area, disruptive technologies are new, with an initial applicability potential unclear and with adaptations to the environment in which they are used. An example of disruptive technology is Web 4.0, an Internet version that will be based on intelligent searches using AI. IoT will play an important role in Web 4.0 technology, along with 
multi-cloud, different forms of computational algorithms (cognitive, edge, invisible, neuromorphic, Web-scale, wearable), blockchain. An impressive number of devices will be intelligently connected via real-time IoT platforms, providing consumers with outstanding features throughout their lifecycle, and benefits businesses in terms of lowering costs and increasing production capacity. Cloud systems will be decentralized and will allow deployment of ubiquitous AI systems, and 5G technology will support the increased security of all these massively distributed systems. 3D printing technology will radically change supply chains and logistics. All of these technologies have a strong disruptive character, and the development of platforms that integrate intelligent functions close to human intelligence is a feasible goal.

Business Process Software is a set of critical applications for computers. At the same time, enterprise software is a collection of customizable programs for an entire organization, requiring specific business and computing skills for implementation. It is meant to serve the entire organization - not just an area or division with a business-specific logic. Every day, employees schedule appointments, request approvals, review documents and workflows, track information, and look for status updates. In many companies, people still do these things manually. Automated activities eliminate concerns about lost emails, errors committed, or a person's presence that depends on an important stage in the business process. Whatever the business process, it can be automated if it is repetitive and involves a number of predictable steps. BPA effectively helps improve communication, minimize costs by more resource allocation, reduce consumables, eliminate manual errors, optimize workflows, establish a clear hierarchy of approval, maintain a steady speed of process by reducing the time required to perform repetitive tasks. It is estimated that a significant percentage of the business processes of companies can be automated. Each industry has different needs for automating business processes. In order to automate as many repetitive processes, businesses are moving towards BPA principles to streamline them. Here are some examples of how different industries can benefit from BPA [3,14,17]: Telecommunications (automates customer service, billing systems, databases, document/workflow management systems and technical support), commerce and industrial production (automates CRM, ESB and ERP systems, warehousing and acquisition activities, production processes and document flow management), banking centers (automation of credit processes, credit and money transfers, integration with other systems automatic banking, card systems and budget management, together with the development of front-and backoffice systems). In addition to simplifying activities, there are many ben efits in implementing BPA/R, including low costs, more efficient use of the workforce, better collaboration, better customer support.

Using a software robot to automate business processes is becoming a widespread practice. On the one hand, managers are beginning to discover the benefits of these solutions, and on the other hand, local suppliers are developing more and more interesting offers. Software robots support employees in achieving a high level of productivity by taking repetitive tasks with no added value. RPA technology can handle any business process involving large volumes of data, from extracting and processing data sets from various documents, transferring files and folders to different locations, reading and writing to / from databases or applications, etc. A software robot can automate, for example, obtaining monthly activity reports, entering invoices and orders, processing holiday requests, or completing customer forms. The most common scenarios for the use of software robots are in the financial and accounting fields (payment and invoice processing, registry completions, database retrievals) human resources management (timing, payroll, employee data processing) and logistics. However, the applicability is much broader, in any field where workflows are repetitive, involves a large amount of data and respects certain templates, but without a high level of complexity. Also, the use of software robots is also recommended for companies with multiple applications on different technologies where integration through connector development generates high costs and data processing times are high. Labor is becoming more and more expensive and harder to find. Therefore, companies need a smart tool that reduces gross workload and repetitive tasks. They block employees' creativity and affect their productivity. The financial sector will be the largest beneficiary of BPA/R, solutions providing financial institutions with added value due to the accuracy and speed with which they perform their tasks. Many financial institutions look to complete process automation, but this is not possible. There are many potential opportunities not only for classical automation but also for the integration of AI elements. For example, a financial sector employee who wishes to analyze market trends to run an investment can receive information through intelligent solutions that will help him make the best decision $[36,41]$.

BPA is becoming a major element of investment, and many European organizations are beginning to focus on the next generation of AI technologies for personalization or prediction purposes. There is currently a business consensus that traditional classical CPU architecture does not meet the mission of running ML ML algorithms. That's why the attention of many goes to the graphics processors to get the performance that ML needs. Despite this phenomenon at European level, examples of how the near future will look are also offered by giants in technology. And that's because Web giants, which require even higher levels of performance, are already starting to develop custom AI acceleration hardware. Google has announced the development of a personalized AI chip three years ago. Google has launched a chip named TPU (Tensor Processing Unit), based on a proprietary integrated circuit used by the company and built specifically for ML processes, being tailored specifically for the company's TensorFlow Learning Module, a neural network based module DNN). Custom chips are extraordinarily expensive and limited in usage because they are designed for specific applications, 
such as the TensorFlow DNN module for Google. Another giant of technology, Microsoft, has accelerated AI in its Azure cloud environment by using a so-called Field Programmable Gate Array (FPGA), with similar performance, in Microsoft's view, of custom chips. Chosen Brainwave, Microsoft's process architecture acceleration process is based on Intel technology to enable scientists and developers to accelerate real-time IA calculations. For now, GPUs are the entry point for most organizations looking to install hardware to accelerate ML algorithms. According to analysts, such units now fit the need for neural network training for IA applications. As neural networks are created from large numbers of identical neurons, they are parallel in nature. Such parallelism fits perfectly into the architecture of GPUs. Where regulations or size of data sets make this impossible, organizations have already begun building their own GPU-based GPs. According to analysts, in the case of ML performance processes, data will have to stay in the cloud environment. Cloud computing services are increasingly present on the market and can increase productivity and team work by providing accessibility from anywhere in the Internet. Internet connections are also becoming increasingly powerful and ubiquitous, and the number and variety of connected devices is steadily increasing. Cloud services are usually sold according to their needs and usability, and thus offer a degree of flexibility that is extremely useful for startups. Users can expand the portfolio of services used in the company's rapid growth or when they want to start new business activities remotely and quickly, according to current needs. This is possible without investing in new hardware and software solutions and lower operating costs. The idea of sharing the computing power of remote computers and servers dates back many years. Over time, cloud computing has become a fully-developed activity, services have become more specialized, and service providers have begun to rent the unlimited computing power of their data centers for applications and programs. Based on the usage ratio of a cloud service provider, there are three basic levels of cloud computing: Software as a Service (SaaS), Platform as a Service (PaaS), and Infrastructure as a Service (IaaS). Cloud services from a vendor are called public cloud services; if a company builds an internal cloud, that is, in its environment, then it is a private cloud service. The new technologies also support a mixed mode - the socalled hybrid cloud service that uses a private cloud and a host of IT services from external sources, that is, from external providers. As we mentioned, cloud computing is an evolving consequence of virtualization, a division of a physical server (memory, storage, or network) into a number of drives that work separately with their own operating systems and power allocated allocation. When virtualization of the company's servers reaches a significant level, say about three-quarters of hardware capacity, the situation starts to resemble cloud computing and all the benefits it implies, especially efficient capacity management. Moving into the Cloud presents a number of key benefits: besides cost reduction and zero initial investment, it is a quick startup of services, including testing the solution. Configuring cloud services is based on business requirements of the customer who only pay monthly for resources used as licenses, dedicated storage, and performance.

\section{Conclusions}

DT is a challenge for leadership, management and human resources. Now that most companies have understood what digitization means and why it's needed, we are stepping into an era where many people have initiated processes of innovation and boosting change within their own organizations. As we advance over time, it is less technological adoption and more strategic planning in close correlation with the incorporation of the main technologies to achieve a competitive advantage. There is still much to do in terms of capturing data in a structured way and extracting the right conclusions from this process. With better processing power that can improve learning, all leaders need to improve their efficiency in how data is used, and this can be achieved through ML and AI. As organizations have realized that private cloud platforms, public cloud, or data centers are not the best option, they have begun combining them. This is how cloud platforms have progressed steadily to meet the growing needs of organizations. This process incorporates all the elements, from cloud to network storage, security, and application deployment. There is a growing need for increased flexibility in terms of high volume of tasks, as well as the ability to quickly expand and expand according to business needs. The developing of the 'ITaaS' concept involves services that enable scalability, shorter acquisition cycles and improved agility, and gives companies the opportunity to be as competitive as the market, but without paying the market price. Blockchain allows transactional transparency across a wide range of commercial functions: almost any decentralized trading process can benefit from blockchain accounting records. Blockchain offers as a service are multiplying, helping companies build apps based on blockchains alone. The aim of all these technological mixes based on AI is to increase the use of data in a right formula as close as possible to the global competitiveness of companies. The AI domain has been experiencing a true evolution due to the processing power that has grown and the size of the computing systems has fallen in size. Due to the evolution, intelligent agents have also improved, becoming more and more intuitive due to different learning techniques. It helps to process a huge amount of data and deliver fast and efficient solutions. Organizations collect information to build their strategy, while others are already developing pilot projects or adopting solutions of this kind. Such intelligent systems have begun to defend in the marketplace and help companies communicate more easily with consumers, develop marketing strategies, and rapidly anticipate consumer needs. 
InA applications are numerous and can be of real use in improving consumer experience. InA will have profound implications at the economic and social level, will reshape new business models, require new algorithms that are ever closer to human intelligence, will generate new jobs based intensively on innovation and maintain a level of action slightly equal to intelligent cars. In business, BPA has a wealth of uses, ranging from fraud detection and improving consumer experience by estimating the services it needs. It can be used in the management of labor and production processes, being able to anticipate certain errors before they occur and to support predictive maintenance. InA is currently, at least in the business field, quite poorly implemented at the level of the companies, focusing especially on getting profits in the classic version. Globally, we can speak of a degree of automation of about 15\%, among countries that exceed this average being USA, France, Germany, UK and India. As InA's objectives, we can recall increasing product quality, increasing labor productivity, increasing customer satisfaction. The links between InA, IA, innovation, economic growth and employment are complex. Some perceive the introduction of new technologies in all company functions as destructive to a large number of workstations. Most tasks performed by robots on production lines or softwares illustrate this perception, even if they can reduce difficulties or repeat certain tasks, improving the added value of the workstation. Others consider that the effects of innovation are very different depending on the period considered, the positive effects on the general occupation of disruptive technology can be measured many years later. At micro level, new CTs can create businesses or activities. Increasing the economic potential and business efficiency the CTs are extremely important in the coming years. For some countries, developing and deploying these technologies on a large scale is a desideratum that tends to materialize in each of the coming years. Using these technologies will develop cognitive enterprises whose activities will be based on a number of specialized intelligent platforms. These platforms will have IoT or WoT input streams, will be supported by multicloud architectures, they will be centered on their business processes and interaction with people. Adaptive learning capacities will be present in their various forms.

\section{Acknowledgement}

This paper was supported by the project "Excellence, performance and competitiveness in the Research, Development and Innovation activities at "Dunarea de Jos" University of Galati", acronym "EXPERT", financed by the Romanian Ministry of Research and Innovation in the framework of Programme 1 - Development of the national research and development system, Sub-programme 1.2 - Institutional Performance - Projects for financing excellence in Research, Development and Innovation, Contract no. 14PFE/17.10.2018.

\section{References}

1. AbuShawar, B., Atwell, E. (2015): Alice Chatbot: Trials and Outputs. Computación y Sistemas, Vol. 19(4), pp. 625-632

2. Agrawal, A., Gans, J., Goldfarb, A. (2017): How AI Will Change the Way We Make Decisions. Harvard Business Review. 26.7.2017. Available: https://hbr.org/2017/07/how-ai-will-change-the-way-we-make-decisions

3. Aguirre, S. \& Rodriguez, A. (2017): Automation of a Business Process Using Robotic Process Automation (RPA): A Case Study. Communications in Computer and Infor-mation Science, Vol. 742, pp.65-71

4. Anagnoste, S. (2017): Robotic Automation Process -The next major revolution in terms of back office operations improvement Proceedings of the 11th International Conference on Business Excellence

5. Andrews, W., F. Karamouzis, K. Brant, M. Revang, M. Reyn-olds, J. Hare, and D. Berman. (2016): Predicts 2017: Artificial Intelligence.” Gartner Research Report, ID: G00317025

6. Andrews, W., D. Berman, A. Linden, and T. Austin. (2017): Artificial Intelligence Primer for 2017. Gartner Research Report, ID: G00318582, February 3, 2017

7. Anifowose, F.A. (2013): Artificial intelligence application in reservoir characterization and modeling: Whitening the black box. Society of Petroleum Engineers -Saudi Arabia Section Young Professionals Technical Symposium 2011, pp. 45-57

8. Asatiani, A., Penttinen, E. (2016): Turning robotic process automation into commercial success -Case OpusCapita. Journal of Information Technology Teaching Cases, 1 No-vember 2016,Vol 6(2), pp. 67-74

9. Asatiani, E., Kasslin, H., Penttinen, E. (2018): How to Choose Between Robotic Process Automation and Back-end System Automation Twenty-Sixth Conference on Information Systems (ECIS2018). Portsmouth, UK

10. Asbjørn, F., Brandtzæg, P., B. (2016): Chatbots and the New World of HCI. Interactions, Vol. 24(4), pp. 38-42

11. Austin, T. (2017): Smart Machines see Major Breakthroughs after Decades of Failure. Gartner Research Report, ID: G00291251, January 4

12. Austin, T., M. Hung, and M. Revang. (2016): Conversational AI to Shake up your Technical and Business Worlds. Gartner Research Report, ID: G00315689, September 30

13. Bergstein, B. (2017): The Great AI Paradox. MIT Technology Review: https://www.technologyreview.com/s/609318/the-great-aiparadox/

14. Bernstein, A. (2016): Globalization, Robots, and the Future of Work: An Interview with Jaffrey Joerres. Harvard Business Review, October 2016, 74-79

15. Brynjolfsson, E., Mitchell, T. (2017): What can machine learning do? Workforce implications: Profound change is coming, but roles for humans remain. Science, Vol. 358(6370), pp. 1530-1534

16. Burgess, A. (2017): The executive guide to artificial intelligence: how to identify and implement applications for AI in your organization. Springer International Publishing $A G$

17. Burnett, S., Jain, A., Modi, A. (2017): Rise of Automation in P\&C Insurance. Everest Group. 8.2.2017.67

18. Burnet, S., Modi, A. (2017): RPA Futures -Accelerated and Intelligent automation. Everest Group. 8.12.2017

19. Burnett, S. (2017): Automating Content-Centric Processes with Artificial Intelligence (AI). Everest Group. 8.12.2017.

20. Capghemini, (2019): A game changer for business operations, Intelligent Automation, Special Edition https://www.capgemini.com/wp-content/uploads/2019/02/Intelligent-Automation-Special Edition_web_final.pdf

21. Castelvecchi, D. (2016): Can we open the black box of AI? Nature, Vol. 538(7623), 5 October 2016, pp. 20-23

22. Cambria, E., White, B. (2014): Jumping NLP Curves: A Review of Natural Language Processing Research. IEEE Computational Intelligence Magazine, May 2014 Issue 
23. Campolo, A., Sanfilippo, M., Whittaker, M., Crawford, K. (2017): AI Now 2017 Report. Available: https://ainowinstitute.org/AI _Now_2017_Report.pdf

24. Craig, A., Lacity. M., Willcocks, L. (2015): Robotic Process Automation at Xchanging. The Outsourcing Unit Working Research Paper Series. 15/03

25. CISCO, (2018): Transforming Business with Artificial Intelligence, http://innovationinsider.com.br/wp-content/uploads/ 2018/09/60280-MITSMR-BCG-Report-2018.pdf

26. Deloitte Insights, (2018): Tech Trends: The Symphonic Enterprise. https://www2. deloitte.com/content/dam /insights/us /articles/Tech-Trends-2018/4109_TechTrends-2018_FINAL.pdf.

27. Felix Weber, Reinhard Schütte (2019): A Domain-Oriented Analysis of the Impact of Machine Learning-The Case of Retailing. Big Data Cogn. Comput. 2019, 3, 11; doi:10.3390/bdcc3010011, Published : 24 January 2019

28. Higginbotham, S. (2016): Here's Why Elon Musk and Everyone Else is Betting on AI. Fortune, December 16, 2015

29. Hoffman, R. (2015): Using Artificial Intelligence to Set Information Fr e e. "MIT Sloan Management Review 58, no. 1

30. Huang, A. H., R. Lehavy, A. Y. Zang, and R. Zheng, (2018): Analyst information discovery and interpretation roles: A topic modeling approach, Management Science. 64, 2473-2472

31. IBM Institute for Business Value (2019): The Cognitive Enterprise: Reinventing your company with AI. Seven keys to success. https://www.ibm.com/events/in-en/think/skills-summit/assets/cog_enterpris e_reinv_your_company_research_insights_v2

32. John-David Lovelock, Susan Tan, Jim Hare, Alys Woodward, Alan Priestley (2018): Forecast: The Business Value of Artificial Intelligence, 2017-2025, Gartner, http://k1.caict.ac.cn/yjts /qqzkgz/zksl/201805 /P020180504572266109739.pdf

33. Lacity, M., Willcocks, L. (2016c): Robotic Process Automation: The Next Transformation Lever for Shared Services. The Outsourcing Unit Working Research Paper Series, London School of Economics and Political Science, January 2016. p. 35.69

34. Lacity, M., Willcocks, L., Craig, A. (2015): The IT Function and Robotic Process Automation. The Outsourcing Unit Working Research Paper Series, London School of Economics and Political Science, October 2015. p. 38.

35. Liang, H., Dickerson, C.E., Champion, D., Battersby, D. (2016): A Framework for Technology Assessment from Different Scientific Viewpoints: Preliminary Report. 11th System of Systems Engineering Conference (SoSE), Kongsberg, pp. 1-5.

36. Kaplan, J. (2016): Artificial Intelligence: What Everyone Needs to Know. Oxford University Press.

37. Kearney, C., \& Liu, S. (2014): Textual sentiment in finance: A survey of methods and models. International Review of Financial Analysis, 33, 171-185

38. Kawa, A., Pieranski, B. Zdrenka, W. (2018): Dynamic Configuration of Same-Day Delivery in E-commerce. In Modern Approaches for Intelligent Information and Database Systems; Sieminski, A., Ed.; Springer: Berlin, Germany, 2018; pp. $305-315$.

39. Klotz, F. (2016): Are You Ready for Robot Colleagues?” MIT Sloan Management Review Digital, http://sloanreview.mit.edu /article/are-you-ready-for-robot-colleagues/

40. KPMG, (2018): Rethinking the value chain, A study on AI, humanoids and robots, Trend one, Artificial Intelligence: Possible Applications and Development Scenarios to 2040 https://assets.kpmg/content/dam/kpmg/xx/pdf/2018/09/rethinking-the-value-chain.pdf

41. Mazilescu V., (2012): A Knowledge Management System Embedded in the New Semantic Technologies. In: New Research in Knowledge Management Technologies, ISBN: 978-953-51-0074-4, Edited by Huei Tse Hou, National Taiwan University of Technology, https://www.intechopen.com/books/new-research-on-knowledge-management-technology

42. MMC VENTURES, (2019): The State of AI 2019: Divergence, In partnership with Barclays. Report design by Aviary Creative, https://www.mmcventures.com/wp-content/uploads/2019/02/The-State-of-AI-2019-Divergence.pdf

43. Philippe Vannier, (2019): Which technologies will power your business tomorrow? Look Out 2020+ Technology Radar: key technologies set to impact you in the coming years., https://atos.net/content/mini-sites/look-out-2020/assets/pdf /ATOS_LOOK\%20OUT_TECH\%20TRENDS.pdf

44. Pierdicca, R.; Liciotti, D.; Contigiani, M.; Frontoni, E.; Mancini, A.; Zingaretti, P. (2015): Low cost embedded system for increasing retail environment intelligence. In Proceedings of the 2015 IEEE International Conference on Multimedia \& Expo Workshops (ICMEW), Turin, Italy, 29 June-3 July 2015

45. Sam Ransbothham, Philipp Gerbert, Martin Reeves, David Kiron, and Michael Spira (2018): Artificial Intelligence in Business Gets Real, MIT Sloan Management Review, Research Report, in collaboration with BCG Henderson Institute, Pioneering Companies Aim for AI at Scale, https://www.pega.com /system/files /resources/2018-05/AI-in-Business.pdf

46. Shabbir J., Anwer T., (2018): A Survey of Deep Learning Techniquesfor Mobile Robot Applications,"ArXiv e-prints, Mar. 2018, https://arxiv.org/pdf/1803.07608.pdf

47. Singh N, Kapoor A., (2015): Cloud Hopfield neural network: Analysis and simulation. Advances in Computing, Communications and Informatics (ICACCI), IEEE. pp 203-209

48. Soni N, Sharma E K, Singh N, Kapoor A., (2018): Impact of Artificial Intelligence on Business. Digital Innovations, Transformation, and Society Conference 2018 (Digits 2018). pp:10

49. Suponenkovs, A., Sisojevs, A., Mosans, G., Kampars, J., Pinka, K., Grabis, J., Taranovs, R. (2017): Application of image recognition and machine learning technologies for payment data processing review and challenges. In Proceedings of the 20175 th IEEE Workshop on Advances in Information, Electronic and Electrical Engineering (AIEEE), Riga, Latvia, 24-25 November 2017.

50. The Royal Society, (2018): The impact of Artificial Intelligence on work. An evidence synthesis on implications for individuals, communities, and societies, British Academy, for the humanities and social sciences, ISBN: 978-0-85672-626-2, https://www.thebritishacademy.ac.uk/sites/default/files/AI-and-work-evidence-synthesis.pdf 